\title{
NFIB promotes the migration and progression of kidney renal clear cell carcinoma by regulating PINK1 transcription
}

\author{
Ninghua Wang ${ }^{\text {Corresp., } 1}$, Jing Yuan ${ }^{2}$, Fei Liu ${ }^{2}$, Jun Wei ${ }^{2}$, Yu Liu ${ }^{2}$, Mei Xue ${ }^{2}$, Rui Dong ${ }^{2}$ \\ 1 Department of Urology, Hanyang Hospital, Wuhan, Hubei, China \\ 2 Department of Urology, Hanyang Hospital, Wuhan, Hubei, china \\ Corresponding Author: Ninghua Wang \\ Email address: wnh120150930@163.com
}

Kidney renal clear cell carcinoma (KIRC) is the most common and aggressive type of renal cell carcinoma. Due to high mortality rate, high metastasis rate and chemical resistance, the prognosis of KIRC patients is poor. Therefore, it is necessary to study the mechanisms of KIRC development and to develop more effective prognostic molecular biomarkers to help clinical patients. In our study, we used The Cancer Genome Atlas (TCGA) and Gene Expression Omnibus (GEO) databases to investigate that the expression of nuclear factor I $B(N F I B)$ is significantly higher in KIRC than in adjacent tissues. Moreover, NFIB expression levels are associated with multiple clinical pathological parameters of KIRC, and KIRC patients with high NFIB expression have poor prognosis, suggesting that NFIB may play vital roles in the malignant development of KIRC. Further studies demonstrated that NFIB could promote the progression and metastasis of KIRC and participate in the regulation of PTEN induced kinase 1 (PINK1). Furthermore, we used chromatin immunoprecipitation (ChIP) experiments to confirm that NFIB binds to the PINK1 promoter and regulates its expression at the transcriptional level. Further experiments also confirmed the important roles of PINK1 in promoting the development of tumors by NFIB. Hence, our data provide a new NFIB-mediated regulatory mechanism for the tumor progression of KIRC and suggest that NFIB can be applied as a new predictor and therapeutic target for KIRC. 
1 NFIB promotes the migration and progression of

2 kidney renal clear cell carcinoma by regulating PINK1

3 transcription

4 Ninghua Wang ${ }^{1}$, Jing Yuan ${ }^{1}$, Fei Liu ${ }^{1}$, Jun Wei ${ }^{1}$, Yu Liu ${ }^{1}$, Mei Xue ${ }^{1}$ and Rui Dong ${ }^{1}$

$5{ }^{1}$ Department of Urology, Hanyang Hospital, Wuhan University of Science and Technology,

6 Wuhan, Hubei, China

7 Correspondence Author:

8 Dr Ninghua Wang, Department of Urology, Hanyang Hospital, Wuhan, Hubei, China

9 E-mail address: wnh120150930@163.com

\section{Abstract}

Kidney renal clear cell carcinoma (KIRC) is the most common and aggressive type of renal cell carcinoma. Due to high mortality rate, high metastasis rate and chemical resistance, the prognosis of KIRC patients is poor. Therefore, it is necessary to study the mechanisms of KIRC development and to develop more effective prognostic molecular biomarkers to help clinical patients. In our study, we used The Cancer Genome Atlas (TCGA) and Gene Expression Omnibus (GEO) databases to investigate that the expression of nuclear factor I B (NFIB) is significantly higher in KIRC than in adjacent tissues. Moreover, NFIB expression levels are associated with multiple clinical pathological parameters of KIRC, and KIRC patients with high NFIB expression have poor prognosis, suggesting that NFIB may play vital roles in the malignant development of KIRC. Further studies demonstrated that NFIB could promote the progression and metastasis of KIRC and participate in the regulation of PTEN induced kinase 1 (PINK1). Furthermore, we used chromatin immunoprecipitation (ChIP) experiments to confirm that NFIB binds to the PINK1 promoter and regulates its expression at the transcriptional level. Further experiments also confirmed the important roles of PINK1 in promoting the development of tumors by NFIB. Hence, our data provide a new NFIB-mediated regulatory mechanism for the tumor progression of KIRC and suggest that NFIB can be applied as a new predictor and therapeutic target for KIRC.

Key words: NFIB, PINK1, kidney renal clear cell carcinoma, metastasis, progression

\section{Introduction}

Renal cell carcinoma (RCC) is one of the most common primary malignant tumors of the kidney, among which kidney renal clear cell carcinoma (KIRC) is the most common and aggressive type, accounting for approximately $60 \%-70 \%$ of RCC histological subtypes (Rini, Campbell \& Escudier, 2009). An estimated 63,990 new cases and 14,400 deaths (including 4940 women and 9470 men) were caused by KIRC in 2017 worldwide (Pang et al., 2018). Due to high mortality rate, high metastasis rate and chemical resistance, the prognosis of KIRC patients is poor (Chaffer \& Weinberg, 2011). Genomic studies have identified several genes involved in the 
progression of KIRC, including BRCA1-associated protein-1 (BAP1), polybromo 1 (PBRM1), SET domain 2 (SETD2) and von-Hippel Lindau tumor suppressor (VHL) (Piva et al., 2015; Nargund et al., 2017). Patients with KIRC are usually treated with standard surgical resection. However, recent studies have shown that the quality of life of KIRC patients varies greatly, and $30 \%$ of local tumor patients eventually develop metastases (Hutson \& Figlin, 2007). Tumor metastasis results in $90 \%$ of cancer-related deaths and poor outcomes for all cancer patients, including KIRC (Moch et al., 2016). More unfortunately, after receiving medical treatment, most KIRC patients eventually develop progressive disease due to intrinsic resistance or acquired resistance (Hsieh et al., 2017). Therefore, it is necessary to study the mechanisms of KIRC development and to develop more effective prognostic molecular biomarkers to help clinical patients.

Site-specific DNA binding proteins in the nuclear factor I (NFI) family play an important role in DNA replication and in the regulation of transcription of a variety of cellular genes (Gronostajski, 2000). The family consists of four genes (NFIA, NFIB, NFIC and NFIX) encoding proteins that interact with DNA as homologous dimers or heterodimers (Kruse \& Sippel, 1994). Transcriptome analysis has shown that NFIB is generally more highly expressed in human tumor tissues, which supports the important role of NFIB in tumor biological processes (GTEx, 2015). Wu et al. noted that NFIB is associated with key molecular events that drive nonsmall cell lung cancer metastasis (Wu et al., 2017). In addition, studies have shown that NFIB directly promotes EZH2 expression and mediates highly invasive and migratory phenotypes in melanoma (Fane et al., 2017). Although NFIB has an established role as an oncogene in nonsmall cell lung cancer and melanoma, it has also been reported that NFIB has a tumor suppressor function in some cancer types. Vo TM et al. demonstrated that NFIB exhibits tumor suppressor activity in glioblastoma, the expression level of NFIB was inversely correlated with astrocytoma tumor grade, and the ectopic expression of NFIB significantly inhibited tumor growth in vivo (Vo et al., 2019). However, to date, no previous studies have reported the roles of NFIB in the malignant development of KIRC and its mechanisms in the tumor growth and metastasis of KIRC.

In our study, we found that NFIB was highly expressed in KIRC through The Cancer Genome Atlas (TCGA) and Gene Expression Omnibus (GEO) data analysis. To further validate our results, we used cell and clinical samples for repeated validation. In vitro, we found that NFIB could significantly promote the proliferation and migration of KIRC cells, which has greatly attracted our interest. Using bioinformatics analysis, we found that PTEN-induced putative kinase 1 (PINK1) may be a downstream site of NFIB; thus, we further demonstrated the promotion roles of PINK1 in NFIB expression and found a binding site for NFIB on the PINK1 promoter using a ChIP assay. In conclusion, we confirmed that NFIB can promote the proliferation and metastasis of tumor cells by transcriptionally regulating the expression of PINK1 in KIRC.

\section{Materials \& methods}

\section{Patients and clinical samples.}


77 Thirty pairs of clinical tissue samples of KIRC were collected from patients undergoing surgery

78 at the Department of Urology, Hanyang Hospital (Wuhan, China) from September 2015 to December 2015. The diagnosis of KIRC was confirmed by the original histopathological reports. The tissue samples were rapidly fixed in formalin for paraffin embedding. All clinical samples were collected with written informed consent to participate in this study. The study was approved by the Ethics Committee of the Academic Medical Center of Wuhan University of Science and Technology (No. 2020-IEC1453).

\section{Cell culture.}

The source of the tubular epithelial cell line (HKC-5; BNCC100598) and KIRC cell lines (LoMet-ccRCC and 786-0) were obtained from the BeNa Culture Collection (Beijing, China). Cell culture methods were performed as previously described (Yuan et al.,2019) .

\section{Transfection.}

The siRNA and normal control (NC) siRNAs for NFIB and PINK1 were purchased from RiboBio (Guangdong, China). The sequences of siRNAs are as follows: NFIB sense: 5'CCAGGUGGUGAAGAAUCUATT-3', antisense: 5'-UAGAUUCUUCACCACCUGGTT-3'; PINK1 sense: 5'- CAAGACUUGGAGCUAAAAATT-3', antisense: 5'UUUUUAGCUCCAAGUCUUGTT-3'. The overexpression plasmids of NFIB and PINK1 were constructed and cloned into the CV061 vector (GeneChem, Shanghai, China) between the EcoRI and HindIII sites. Once the cells reached 30-40\% confluence, LoMet-ccRCC and 786-0 cells were transfected using Lipofectamine 3000 reagent (Invitrogen, MA, USA) according to the manufacturer's instructions. Subsequent treatments were performed after the cells were cultured for an additional 48 hours.

\section{CRISPR-Cas9}

NFIB-knockout cells were generated using CRISPR-Cas9 gene editing, and the sgRNA targeting NFIB gene was cloned into GV371-U6-NFIB sgRNA-SV40-EGFP and GV371-CMV-hSpCas9SV40-Puro, respectively. The sgRNA sequence is as follows: GGGAGTGTCTCCTGCCGAGA. In addition, LV-sgRNA and LV-Cas9 lentiviruses were also produced (GeneChem, Shanghai, China). LV-Cas9 was seeded in 786-0 cells with a MOI of 2 after 3 days of puromycin selection with a final concentration of $5 \mu \mathrm{g} / \mathrm{ml}$. LV-sgRNA was then seeded, and another 3 days later, the cells were harvested for further detection.

\section{qRT-PCR.}

Quantitative real-time reverse transcription polymerase chain reaction (qRT-PCR) was performed as previously described (Yuan et al.,2019). The following primers were used: NFIB forward, 5'-GTAATTAGAGCTGTGACA-3'; NFIB reverse, 5'-TGTATGCCATCTAGTGGAT3'; PINK1 forward, 5'-CGTGGCGAGCTGTAATTGAC-3'; PINK1 reverse, 5'GGCTCCGCAGACGTTATCTA-3'; GAPDH forward, 5'-GAAGGTGAAGGTCGGAGTC-3'; and GAPDH reverse, 5'-GAAGATGGTGATGGGATT-3'. All reactions were performed in triplicate. The relative expression levels of the genes were calculated using the $2^{-\Delta \Delta \mathrm{Cq}}$ method.

\section{Western blotting.}

Total protein lysates from the cultured cells were extracted using a lysis buffer containing 
117 proteinase inhibitors (Sigma-Aldrich, Darmstadt, Germany). Proteins were denatured, separated 118 in 10\% polyacrylamide gels, and transferred to polyvinylidene fluoride (PVDF) membranes for 119 probing with the following primary antibodies: anti-NFIB (1:500, Abcam, ab186738,

120 Cambridge, UK), PINK1 (1:500, Abcam, ab216144, Cambridge, UK) and anti-GAPDH (1:2000,

121 Proteintech, 10494-1-AP, Wuhan, China). For detection, anti-rabbit secondary antibodies

122 conjugated to horseradish peroxidase (1:3000, Proteintech, Wuhan, China) were used. Band

123 signals were visualized by an enhanced chemiluminescence detection kit (Meilunbio, Dalian,

124 China). All western blotting was performed in triplicate.

125 Cell proliferation assay.

126 Cells were seeded into 96-well plates at a density of $3 \times 10^{3}$ cells per well and observed for 1 to 1275 days. Then, $20 \mu \mathrm{l}$ of MTT $(5 \mathrm{mg} / \mathrm{mL})$ was added to each well, and the media were replaced 128 with $200 \mathrm{~mL}$ of DMSO (Sigma) after incubation for 4 hours. When the crystals were completely 129 dissolved, a microplate reader was used to determine the resulting absorbance at $570 \mathrm{~nm}$. All 130 MTT assays were performed in triplicate.

\section{Colony formation assay.}

132 Cells of treatments and experimental groups were respectively seeded into six-well plates at a

133

134

135

136

137

138

139

140

141

142

143

144

145

146

147

148

149

150

151

152

153

154

155

density of 500 cells per well and cultured with complete medium at $37^{\circ} \mathrm{C}$ in $5 \% \mathrm{CO}_{2}$ for 15 days. The colonies were then stained with $1 \%$ crystal violet (Sigma-Aldrich, Darmstadt, Germany) for $30 \mathrm{~min}$ and counted. All experiments were performed in triplicate.

\section{Migration and wound-healing assay.}

Transwell chambers (Beaverbio, Jiangsu, China) were used to assess KIRC cell metastasis ability. Suspensions of $8 \times 10^{4}$ cells in $200 \mu$ of FBS-free medium (Sigma-Aldrich, Darmstadt, Germany) were added to the top chamber, and medium containing 20\% FBS (Sciencell, CA, USA) was added to the bottom chamber to serve as the chemotaxin. The cells were cultivated at $37^{\circ} \mathrm{C}$ in $5 \% \mathrm{CO}_{2}$ for 48 hours, and the cells that failed to migrate were wiped off the top of the membranes. The migrated cells attached to the bottom chamber were fixed and stained. Cells from five random fields were quantified by light microscopy. To determine the invasion ability of KIRC cells, invasion assay was performed by transwell migration chambers coated with Matrigel (Sigma).

For the wound-healing assay, the cells were seeded into 12-well plates (Beaverbio, Jiangsu, China) and grown to $80-90 \%$ confluence at $37^{\circ} \mathrm{C}$ in $5 \% \mathrm{CO}_{2}$. The cell monolayers were prepared with a $200 \mu \mathrm{l}$ pipette tip and cultured in serum-free medium. Cell migration was assessed by microscopy at 0 and 48 hours and objectively analyzed with ImageJ 1.8.0. All experiments were performed in triplicate.

\section{Immunohistochemical.}

Paraffin-embedded clinical tissues were cut into 5- $\mu \mathrm{m}$-thick sections and placed on glass slides.

The tissue sections were deparaffinized and subjected to antigen retrieval using $0.01 \mathrm{~m}$ citric acid buffer ( $\mathrm{pH} \mathrm{6.0)}$ ) for $15 \mathrm{~min}$ and incubated overnight at $4{ }^{\circ} \mathrm{C}$ with primary antibodies against NFIB (1:200, Abcam, ab186738, Cambridge, UK). After three washes with Tris-buffered saline, the

Peer) reviewing PDF | (2020:07:51399:2:0:NEW 28 Dec 2020) 
156

157

158

159

160

161

162

163

164

165

166

167

168

169

170

171

172

173

174

175

176

177

178

179

180

181

182

183

184

185

186

187

sections were incubated with a horseradish peroxidase-conjugated secondary antibody (1:100, Boster) for 1 hour at room temperature. The immunohistochemical (IHC) staining results were evaluated by two independent observers. When staining on the nucleus and $\geq 30 \%$ positive cells in the section, immunohistochemical staining of NFIB was estimated to be positive.

\section{Chromatin immunoprecipitation.}

Chromatin immunoprecipitation (ChIP) assays were performed using the SimpleChIP Enzymatic Chromatin IP Kit (CST, \#9005, MA, USA) according to the manufacturer's recommended protocol. Cells were crosslinked with formaldehyde and sonicated to an average size of 150 to $900 \mathrm{bp}$. Then, chromatin extracts were immunoprecipitated using $4 \mu \mathrm{g}$ of monoclonal anti-NFIB antibody (Abcam, ab186738, Cambridge, UK). The purified DNA was subjected to qPCR to amplify the binding sites of the PINK1 promoter region. All ChIP assays were performed in triplicate.

\section{Data acquisition and statistical analysis.}

Raw KIRC data containing mRNA sequencing and clinical information were obtained from the TCGA Genome Data Analysis Center (http:/gdac.broadinstitute.org/runs/analyses_latest/reports/cancer/STAD/). In addition, the KIRC mRNA expression array of GSE83999 and GSE53757 were acquired from the NCBI GEO (Perron et al., 2018; von et al., 2014). The data analysis methods were performed as previously described (Yuan et al., 2019). The R 'limma' Bioconductor package was used to screen the differentially expressed genes (DEGs) between KIRC and adjacent tissues based on the following criteria: Fold change (FC), $|\log 2(\mathrm{FC})|>1$; and false discovery rate (FDR) $<0.05$. Adjusted $\mathrm{P}<0.05$ was used to define a gene as a DEG. Database for Annotation, Visualization and Integrated Discovery (DAVID) v6.8 (david-d. ncifcrf.gov/) was used to analyze functional enrichment among DEGs. In addition, only those Kyoto Encyclopedia of Genes and Genomes (KEGG) pathways with $\mathrm{P} \leq 0.05$ and $\geq 10$ enriched genes were considered significant. The assay data were analyzed by SPSS 13.0 and presented as the mean value \pm standard deviation (SD). Depending on the type of experiment performed, the assay results were analyzed through two-tailed unpaired or paired Student's $t$ test. The relationships between the expression of NFIB and the clinical pathological characteristics of KIRC were analyzed by chi square $\left(X^{2}\right)$ test. The statistical significances between the groups are presented as $P$ values. When $p<0.05$, the results were considered statistically significant.

\section{Results}

\section{NFIB is overexpressed in human KIRC.}

To identify novel genes that potentially drive KIRC tumorigenesis, we performed differential expression analysis using the dataset from TCGA (Table S1) and the datasets from GEO (Fig. $1 \mathrm{~A}$ and Table S2-3). Hierarchical cluster analysis revealed alterations in the mRNA transcript expression between KIRC and paired noncancerous kidney (NK) tissue among the three datasets (Fig. 1B). NFIB was one of the prominently upregulated mRNAs in KIRC tissues compared with NK tissues (Fig. 1B and C). After a literature search, we found that the roles of NFIB in the 
development of KIRC have not been reported, so we aimed to further investigate its roles in driving KIRC tumorigenesis. To confirm these results, we further tested the expression levels of NFIB in cell lines. Compared with normal human renal tubular epithelial cell lines, KIRC cell lines showed significantly increased mRNA expression levels of NFIB (Fig. 1D), and the western blot results were consistent with the results obtained from RT-qPCR (Fig. 1E). In addition, NFIB expression was detected in 30 paired KIRC and NK clinical samples using IHC analysis. The expression level of NFIB was significantly higher in KIRC tissues than in paired NK clinical tissues (Fig. 1F and G). Taken together, these results demonstrate a novel dysregulated gene, NFIB, in KIRC.

\section{NFIB is associated with KIRC progression and worse prognosis.}

To explore the clinical significance of NFIB, we assessed the correlation between the expression levels of NFIB and the individual clinicopathological features of KIRC patients using the dataset from TCGA (Table S4). As shown in Table 1, the results indicated that the overexpression of NFIB was significantly associated with poor histological grade $(\mathrm{P}=0.008)$, pathological stage $(\mathrm{P}=0.011)$, T stage $(\mathrm{P}=0.035)$, lymphatic invasion $(\mathrm{P}<0.001)$ and distant metastasis $(\mathrm{P}=0.019)$. Moreover, we found that high NFIB expression was significantly associated with worse prognosis in patients (Figure 1H, HR: 0.175, 95\% CI: [0.091, 0.336]), and we obtained consistent results (Fig. 1I) with the online data sites GEPIA (http://gepia.cancer-pku.cn) (Tang et al., 2017).

\section{NFIB promotes the proliferation and migration of KIRC cells.}

To investigate the biological roles of NFIB, the mRNA and protein expression of NFIB was upregulated by transfection with plasmids containing the NFIB sequence (NFIB vector) compared to the vector control (NC vector) in the LoMet-ccRCC and 786-0 cell lines (Fig. 2A). The results showed that NFIB overexpression significantly increased the proliferation of the LoMet-ccRCC and 786-0 cell lines (Fig. 2B). We further used colony formation experiments to demonstrate that the overexpression of NFIB promoted the number of tumor cell colonies formed in the LoMet-ccRCC and 786-0 cell lines (Fig. 2C and D). In addition, the migration ability of LoMet-ccRCC and 786-0 cells was significantly increased after NFIB overexpression compared with NC vector transfection in transwell, invasion and wound-healing assays (Fig. 2E, F, G, H, I and J). Additionally, siRNAs against human NFIB (NFIB-si) were applied to knockdown the expression of NFIB in KIRC cells (Fig. 3A). The knockdown of NFIB significantly repressed the proliferation and colony formation of LoMet-ccRCC and 786-0 cells (Fig. 3B, C, D and E). Furthermore, transwell, invasion and wound-healing assays showed that NFIB depletion caused an obvious suppression of the migration ability of KIRC cells (Fig. 3F, G, H, I, J, K, L and M). Therefore, the above results suggest that NFIB might be a promoter of KIRC tumorigenesis.

\section{PINK1 is a potentially target of NFIB.}

To investigate the target genes of NFIB, we first performed functional enrichment analysis of the differentially expressed genes between KIRC and adjacent tissues obtained from three datasets (Fig. 1A and Table S5) using the DAVID online analysis website (https://david.ncifcrf.gov) (Jiao 
235

236

237

238

239

240

241

242

243

244

245

246

247

248

249

250

251

252

253

254

255

256

257

258

259

260

261

262

263

264

265

266

267

268

269

270

271

272

273

274

275

et al., 2012). These genes are significantly enriched in multiple KEGG pathways involved in tumorigenesis (Fig. 4A), such as pathways in cancer $(\mathrm{P}=0.001)$ and MAPK signaling pathway $(\mathrm{P}=0.002)$, associated with tumor cell proliferation, ECM-receptor interaction $(\mathrm{P}<0.001)$ and the calcium signaling pathway $(\mathrm{P}<0.001)$, associated with drug transport and metabolism in tumor cells. Given that NFIB is a transcription factor, we next investigated the possible regulation of NFIB by analyzing the gene promoters enriched in these pathways. The open database JASPAR (http://jaspar.genereg.net) and PROMO (http://alggen.lsi.upc.es/cgi-bin/ promo_v3/promo/promoinit.cgi?dirDB=TF_8.3) were used to predicate the potential NFIB sites on the PINK1 gene promoter. Fortunately, we found three potential binding sites on PINK1 gene promoter for NFIB (Fig. 4B) (Khan et al., 2018; Messeguer et al., 2002), and bioinformatics analysis revealed that PINK1 was enriched in focal adhesion and pathways in cancer (Table S5). Hence, we hypothesized that PINK1 is one of the targets by which NFIB promotes KIRC progression and metastasis.

\section{NFIB regulates PINK1 expression.}

To further verify the role of NFIB, the KIRC cell lines LoMet-ccRCC and 786-0 were infected with NFIB vector or NC vector. Using qRT-PCR analysis, we found that NFIB overexpression significantly induced an increase in the mRNA expression level of PINK1 and that PINK1 expression was not induced by the NC vector (Fig. 4C). Furthermore, we used western blotting to detect the effect of NFIB overexpression on the expression of PINK1 protein. As expected, the protein level of PINK1 was significantly increased by NFIB overexpression compared with NC vector transfection (Fig. 4D). On the other hand, we observed the opposite results in KIRC cell lines after NFIB knockdown. qRT-PCR and western blotting showed that the knockdown of NFIB downregulated the expression levels of PINK1 mRNA and protein (Fig. 4E and F), respectively. As shown in Figure 4B, three putative NFIB transcriptional binding sites in the PINK1 gene promoter region were found via bioinformatics analysis. Next, we performed a ChIP assay in LoMet-ccRCC and 786-0 cells using NFIB-specific antibodies. As shown in Figure 4G, based on the qRT-PCR reads and the ChIP analysis results, we found that the NFIBbound complex was remarkably enriched in PINK1 promoter site 2 but not site 1 or site 3 . These results strongly implied that NFIB promotes KIRC metastasis and progression by regulating the promoter of PINK1.

\section{PINK1 is the critical downstream of NFIB in KIRC.}

To confirm that PINK1 is a key factor through which NFIB promotes KIRC progression, we first conducted a rescue experiment. The cotransfection of PINK1 siRNA with NFIB vector dramatically decreased NFIB-upregulated PINK1 mRNA expression (Fig. 5A and B), and western blotting also obtained consistent results, and the promotion of PINK1 protein expression by NFIB was inhibited by PINK1 siRNA (Fig. 5C). We further examined the effect of PINK1 siRNA on the cell proliferation and progression induced by the overexpression of NFIB using LoMet-ccRCC cells. As expected, under the action of PINK1 siRNA, the increased proliferation and migration abilities of the LoMet-ccRCC cell line after NFIB overexpression were inhibited again (Fig. 5D, E, F, G, H, I, J, K and L). On the contrary, we used CRISPR-Cas9 to knockout NFIB and express exogenous PINK1 under a constitutive expression promoter to observe the 
276 effects of KIRC cell migration, invasion and reproduction. We found that NFIB knockout

277 resulted in a significantly down-regulation of PINK1 (Fig. 6A), and the proliferation and

278 migration ability of KIRC cells was significantly reduced (Fig. 6B, C, D, E, F, G, H, I and J).

279 Then, after the exogenous overexpression of PINK1, the proliferation and migration ability of

280 KIRC cells were significantly restored (Fig. 6A, B, C, D, E, F, G, H, I and J). Therefore, PINK1

281 was the key downstream of abnormal expression of NFIB leading to abnormal biological

282 behavior of KIRC cells. Taken together, these results suggest that PINK1 is a critical target of

283 NFIB and that PINK1 exerts tumor-promoting roles in KIRC.

\section{Discussion}

285

286

287

288

289

290

291

292

293

294

295

296

297

298

299

300

301

302

303

304

NFIB has been reported to play pivotal roles in the tumorigenesis of cancers, including gastric cancer, breast cancer, melanoma, and nonsmall cell lung cancer (Campbell et al., 2018; Fane et al., 2017; Wu et al., 2017; Wu et al., 2018). However, at present, there was no report about the relationship between NFIB mutation and tumor, whether the expression changes of NFIB are related to specific pathological subtypes. Moreover, the functions and mechanisms of NFIB in KIRC tumorigenesis are far from defined. Therefore, it is necessary to further study the role of NFIB in the development of KIRC and the corresponding molecular mechanisms to help to better understand the mechanism of tumorigenesis and to provide novel and valuable targets for drug therapy. A significant new finding in this study is that NFIB plays a role in the proliferation and metastasis of KIRC by regulating PINK1 expression, and ChIP experiments confirmed that NFIB acts primarily through binding to the PINK1 promoter.

We first analyzed TCGA and GEO datasets and found that NFIB is one of the significantly increased genes in KIRC. Further analysis of the correlation between NFIB and KIRC clinical pathological parameters revealed that the high expression of NFIB was significantly associated with KIRC tumors with poor grades, significant metastasis, and worse prognosis. In view of these results, we consider it necessary to further investigate the role of NFIB in KIRC tumor growth and metastasis. With the overexpression of NFIB, the proliferation and migration ability of KIRC tumor cells was significantly improved. Conversely, the knockdown of NFIB significantly reduced the proliferation and metastasis ability of KIRC cells. These results indicate that NFIB may support the progression of KIRC.

To further explore the mechanisms by which NFIB promotes KIRC progression, we performed functional enrichment analysis on DEGs in KIRC in the databases. As shown in Fig. $2 \mathrm{~A}$, we found that multiple pathways are enriched in cell-cell interactions, including cell adhesion molecules, focal adhesion and tight junctions. Moreover, enrichment analysis indicated that multiple pathways are involved in the malignant proliferation of KIRC tumor cells, including the MAPK signaling pathway and pathways in cancer. In addition, the transport functions of the cell membrane play a vital role in the progression of KIRC, including the calcium signaling pathway. Since NFIB is a nuclear transcription factor that plays a major role in the transcriptional regulation of target genes, we wanted to explore the downstream targets of NFIB by analyzing the promoters of genes enriched in the abovementioned pathways. We found that the expression level of PINK1 were indeed altered by the regulation of NFIB expression. 
PINK1 encodes a serine/threonine protein kinase that is primarily localized in the mitochondria (Nguyen, Padman \& Lazarou, 2016). PINK1 was originally found to be upregulated by the overexpression of the tumor suppressor PTEN in HeLa cells, which is thought to protect cells from stress-induced mitochondrial dysfunction (Di Rita et al., 2018). In addition, mutations in PINK1 cause autosomal recessive early-onset Parkinson's disease (Ren et al., 2019). Recent studies have reported the role and mechanism of PINK1 in tumor proliferation, metastasis, apoptosis and tumor resistance (Liu et al., 2018; Zhang et al., 2017). PINK1 interacts with the oncogenic PI3K/Akt/mTOR signaling axis at multiple levels and controls the regulation of cancer survival and growth (Wang et al., 2019). Moreover, studies have indicated that PINK1 has cytoprotective and chemoresistant functions in breast cancer and that PINK1 can be used as a target for breast cancer treatment (Li et al., 2017). Related studies in lung cancer have also shown that the silencing of PINK1 inhibits the migration and invasion of lung cancer cells and that the inhibition of PINK1 enhances the apoptosis rate of cancer cells (Liu et al., 2018). In summary, PINK1 has a strong connection with the malignant development of tumors. Moreover, we used an online analysis site to predict possible binding sites of NFIB on the PINK1 gene promoter (Khan et al., 2018; Messeguer et al., 2002). Furthermore, ChIP experiments further confirmed that "GGGATTCACC" on the PINK1 gene promoter sequence is a binding site of NFIB, which further suggests that PINK1 is directly regulated by NFIB. To demonstrate the critical role of PINK1 in the promotion of KIRC mediated by NFIB, it was necessary to conduct a "rescue" experiment. As expected, when the NFIB vector and the small interfering RNA against PINK1 were simultaneously transfected into the LoMet-ccRCC cell lines, the NFIBinduced promotion of cell proliferation and metastasis was significantly inhibited. Although our studies needs to be further explored in vivo, and the relationship between NFIB and classic oncogenic signaling pathways needs further discussion. However, in conclusion, our study confirmed multiple pathways involved in KIRC progression and further demonstrated the role of NFIB in promoting KIRC progression and metastasis through the transcriptional regulation of PINK1 expression. Moreover, our data analysis found that NFIB expression levels are related to multiple clinical pathological parameters of KIRC and to patient prognosis; thus, NFIB could be used as a potential biomarker to clinically evaluate the severity of KIRC in patients.

\section{Conclusions}

In conclusion, our study demonstrated the role of NFIB in promoting KIRC progression and metastasis through the transcriptional regulation of PINK1 expression. Moreover, we suggested that NFIB could be used as a potential biomarker to clinically evaluate the severity of KIRC in patients.

\section{Acknowledgements}

We sincerely appreciate our team members for providing statistical instructions.

\section{References}


Bergers G, Hanahan D. 2008. Modes of resistance to anti-angiogenic therapy. Nat Rev Cancer 8: 592-603 DOI: $10.1038 / \mathrm{nrc} 2442$.

Campbell TM, Castro MAA, de Oliveira KG, Ponder BAJ, Meyer KB. 2018. ER $\alpha$ Binding by Transcription Factors NFIB and YBX1 Enables FGFR2 Signaling to Modulate Estrogen Responsiveness in Breast Cancer. Cancer Res 78: 410-421 DOI: 10.1158/0008-5472.

Chaffer CL, Weinberg RA. 2011. A perspective on cancer cell metastasis. Science 331: 155964 DOI: $10.1126 /$ science. 1203543

Di Rita A, Peschiaroli A, D' Acunzo P, Strobbe D, Hu Z, Gruber J, Nygaard M, Lambrughi M, Melino G, Papaleo E, Dengjel J, Alaoui SE, Campanella M, Dötsch V, Rogov VV, Strappazzon F, Cecconi F. 2018. HUWE1 E3 ligase promotes PINK1/PARKINindependent mitophagy by regulating AMBRA1 activation via IKK $\alpha$. Nat Commun 9: 3755 DOI: 10.1038/s41467-018-05722-3.

Fane ME, Chhabra Y, Hollingsworth DEJ, Simmons JL, Spoerri L, Oh TG, Chauhan J, Chin T, Harris L, Harvey TJ. 2017. NFIB Mediates BRN2 Driven Melanoma Cell Migration and Invasion Through Regulation of EZH2 and MITF. EBioMedicine 16:63-75 DOI: 10.1016/j.ebiom.2017.01.013.

Gronostajski RM. 2000. Roles of the NFI/CTF gene family in transcription and development. Gene 249: 31-45 DOI: 10.1016/s0378-1119(00)00140-2.

GTEx Consortium. 2015. Human genomics. The Genotype-Tissue Expression (GTEx) pilot analysis: multitissue gene regulation in humans. Science 348: 648-60 DOI: 10.1126/science. 1262110 .

Jiao X, Sherman BT, Huang da W, Stephens R, Baseler MW, Lane HC, Lempicki RA. 2012. DAVID-WS a stateful web service to facilitate gene/protein list analysis. Bioinformatics 28: 1805-6 DOI: 10.1093/bioinformatics/bts251.

Khan A, Fornes O, Stigliani A, Gheorghe M, Castro-Mondragon JA, van der Lee R, Bessy A, Chèneby J, Kulkarni SR, Tan G, Baranasic D, Arenillas DJ, Sandelin A, Vandepoele K, Lenhard B, Ballester B, Wasserman WW, Parcy F, Mathelier A. 2018. JASPAR 2018: update of the open-access database of transcription factor binding profiles and its web framework. Nucleic Acids Res 46: D260-D266 DOI: 10.1093/nar/gkx1126.

Kim SP, Alt AL, Weight CJ, Costello BA, Cheville JC, Lohse C, Allmer C, Leibovich BC. 2011. Independent validation of the 2010 American Joint Committee on Cancer TNM classification for renal cell carcinoma: results from a large, single institution cohort. J Urol 185: 2035-9 DOI: 10.1016/j.juro.2011.02.059.

Kruse U, Sippel AE. 1994. Transcription factor nuclear factor I proteins form stable homo- and heterodimers. FEBS Lett 348: 46-50 DOI: 10.1016/0014-5793(94)00585-0. 
Li GB, Fu RQ, Shen HM, Zhou J, Hu XY, Liu YX, Li YN, Zhang HW, Liu X, Zhang YH, Huang C, Zhang R,Gao N. 2017. Polyphyllin I induces mitophagic and apoptotic cell death in human breast cancer cells by increasing mitochondrial PINK1 levels. Oncotarget 8: 10359-10374 DOI: 10.18632/oncotarget.14413.

Liu L, Zuo Z, Lu S, Wang L, Liu A, Liu X. 2018. Silencing of PINK1 represses cell growth, migration and induces apoptosis of lung cancer cells. Biomed Pharmacother 106: 333-341 DOI: 10.1016/j.biopha.2018.06.128.

Messeguer X, Escudero R, Farré D, Núñez O, Martínez J, Albà MM. 2002. PROMO: detection of known transcription regulatory elements using species-tailored searches. Bioinformatics 18: 333-4 DOI: 10.1093/bioinformatics/18.2.333.

Moch H, Cubilla AL, Humphrey PA, Reuter VE, Ulbright TM. 2016. The 2016 WHO Classification of Tumours of the Urinary System and Male Genital Organs-Part A: Renal, Penile, and Testicular Tumours. Eur Urol 70: 93-105 DOI: 10.1016/j.eururo.2016.02.029.

Nargund AM, Pham CG, Dong Y, Wang PI, Osmangeyoglu HU, Xie Y, Aras O, Han S, Oyama T, Takeda S, Ray CE, Dong Z, Berge M, Hakimi AA, Monette S, Lekaye CL, Koutcher JA, Leslie CS, Creighton CJ, Weinhold N, Lee W, Tickoo SK, Wang Z, Cheng EH, Hsieh JJ. 2017. The SWI/SNF Protein PBRM1 Restrains VHL-Loss-Driven Clear Cell Renal Cell Carcinoma. Cell Rep 18: 2893-2906 DOI: 10.1016/j.celrep.2017.02.074

Nguyen TN, Padman BS, Lazarou M. 2016. Deciphering the Molecular Signals of PINK1/Parkin Mitophagy. Trends Cell Biol 26: 733-744 DOI: 10.1016/j.tcb.2016.05.008.

Pang S, Sun Y, Wu L, Yang L, Zhao YL, Wang Z, Li Y. 2018. Reconstruction of kidney renal clear cell carcinoma evolution across pathological stages. Sci Rep 8: 3339 DOI: 10.1038/s41598-018-20321-4

Perron G, Jandaghi P, Solanki S, Safisamghabadi M, Storoz C, Karimzadeh M, Papadakis AI, Arseneault M, Scelo G, Banks RE, Tost J, Lathrop M, Tanguay S, Brazma A, Huang S, Brimo F, Najafabadi HS, Riazalhosseini Y. 2018. A General Framework for Interrogation of mRNA Stability Programs Identifies RNA-Binding Proteins that Govern Cancer Transcriptomes. Cell Rep 23: 1639-1650 DOI: 10.1016/j.celrep. 2018.04.031.

Piva F, Santoni M, Matrana MR, Satti S, Giulietti M, Occhipinti G, Massari F, Cheng L, Lopez-Beltran A, Scarpelli M, Principato G, Cascinu S, Montironi R. 2015. BAP1, PBRM1 and SETD2 in clear-cell renal cell carcinoma: molecular diagnostics and possible targets for personalized therapies. Expert Rev Mol Diagn 15: 1201-10 DOI: 10.1586/14737159.2015.1068122.

Ren X, Hinchie A, Swomley A, Powell DK, Butterfield DA. 2019. Profiles of brain oxidative damage, ventricular alterations, and neurochemical metabolites in the striatum of PINK1 
knockout rats as functions of age and gender: Relevance to Parkinson disease. Free Radic Biol Med 143:146-152 DOI: 10.1016/j.freeradbiomed.2019.08.008.

Rini BI, Campbell SC, Escudier B. 2009. Renal cell carcinoma. Lancet 373:1119-32 DOI: 10.1016/S0140-6736(09)60229-4.

Schmidinger, Daniel YH, James L, Vincenzo F. Renal cell carcinoma. Nat Rev Dis Primers. 2017 3:17009. DOI: 10.1038/nrdp.2017.9.

Thomas EH, Robert AF. Renal cell cancer. Cancer J 13: 282-6. DOI: 10.1097/PPO.0b013e318156fe69.

Tang Z, Li C, Kang B, Gao G, Li C, Zhang Z. 2017. GEPIA: a web server for cancer and normal gene expression profiling and interactive analyses. Nucleic Acids Res 45: W98W102 DOI: 10.1093/nar/gkx247.

Vo TM, Jain S, Burchett R, Monckton EA, Godbout R. 2019. A positive feedback loop involving nuclear factor IB and calpain 1 suppresses glioblastoma cell migration. $J$ Biol Chem 294:12638-12654 DOI: 10.1074/jbc.RA119.008291.

von Roemeling CA, Radisky DC, Marlow LA, Cooper SJ, Grebe SK, Anastasiadis PZ, Tun HW, Copland JA. 2014. Neuronal pentraxin 2 supports clear cell renal cell carcinoma by activating the AMPA-selective glutamate receptor-4. Cancer Res 74: 4796-810 DOI: 10.1158/0008-5472.CAN-14-0210.

Wang H, Chen S, Zhang Y, Xu H, Sun H. 2019. Electroacupuncture ameliorates neuronal injury by Pink1/Parkin-mediated mitophagy clearance in cerebral ischemia-reperfusion. Nitric Oxide 91:23-34 DOI: 10.1016/j.niox.2019.07.004.

Wu C, Zhu X, Liu W, Ruan T, Wan W, Tao K. 2018. NFIB promotes cell growth, aggressiveness, metastasis and EMT of gastric cancer through the Akt/Stat3 signaling pathway. Oncol Rep 40: 1565-1573 DOI: 10.3892/or.2018.6574.

Wu Y, Zhang J, Hou S, Cheng Z, Yuan M. 2017. Non-small cell lung cancer: miR-30d suppresses tumor invasion and migration by directly targeting NFIB. Biotechnol Lett 39: 1827-1834 DOI: 10.1007/s10529-017-2428-9.

Yuan J, Dong R, Liu F, Zhan L, Liu Y, Wei J, Wang N. 2019. The miR-183/182/96 cluster functions as a potential carcinogenic factor and prognostic factor in kidney renal clear cell carcinoma. Exp Ther Med 17: 2457-2464 DOI: 10.3892/etm.2019.7221.

Zhang R, Gu J, Chen J, Ni J, Hung J, Wang Z, Zhang X, Feng J, Ji L. 2017. High expression of PINK1 promotes proliferation and chemoresistance of NSCLC. Oncol Rep 37: 2137-2146 DOI: 10.3892/or.2017.5486. 


\section{Figure 1}

NFIB was highly expressed and was associated with poor prognosis in KIRC.

(A) Venn plot diagram of differentially expressed genes in TCGA and GEO (GSE83999 and GSE53757) data sets. (B) Top10 mRNA expression profiles (FPKM) and identification of NFIB as differentially expressed between KIRC and normal tissues based on TCGA data set. (C) Volcano plot of differentially expressed mRNAs between KIRC and normal tissues based on TCGA data set. Up, upregulation; Down, downregulation. The levels of NFIB were significantly decreased in KIRC (LoMet-ccRCC, 786-0) compared with the human renal tubular epithelial cell line HKC-5 as indicated by (D) qRT-PCR and (E) western blot assay respectively. The high expression of NFIB in KIRC tissues compared with matched adjacent noncancerous tissues via (F) IHC (Scale bars $=100 \mu \mathrm{m}$ ) and (G) qRT-PCR. Kaplan-Meier curves of KIRC patients stratified based on the expression of NFIB in KIRC tissues (high or low) based on (H) TCGA data set, (I) the online data sites GEPIA (http://gepia.cancer-pku.cn). The data D were presented as means \pm SD of three independent experiments. Values are significant at $* \mathrm{P}<$ 0.05 , as demonstrated by paired Student's t test. 
A

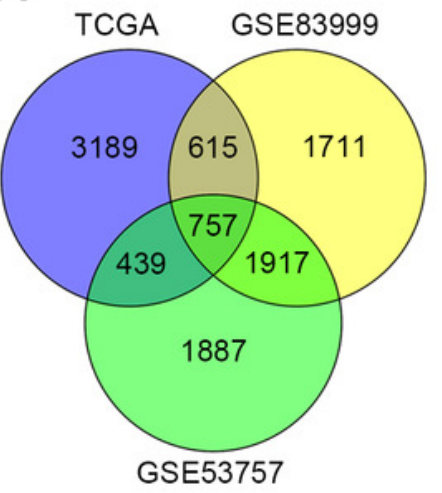

D

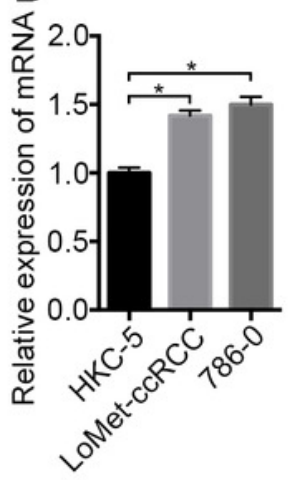

E

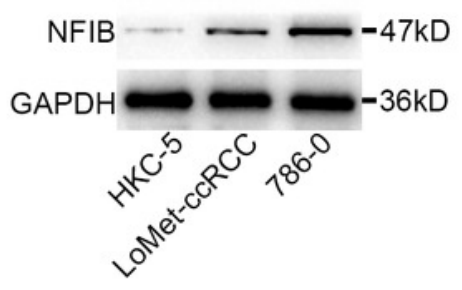

$\mathrm{H}$

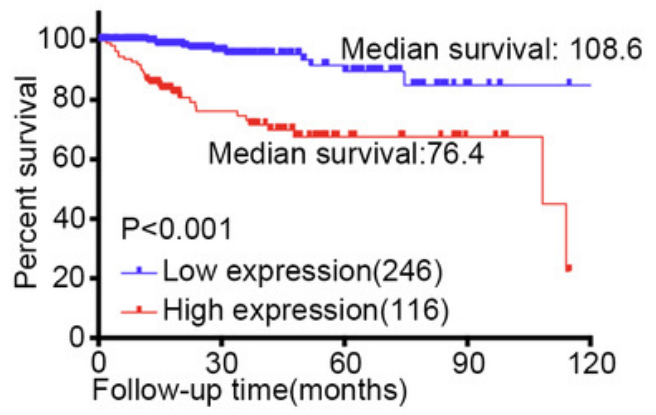

C : Up

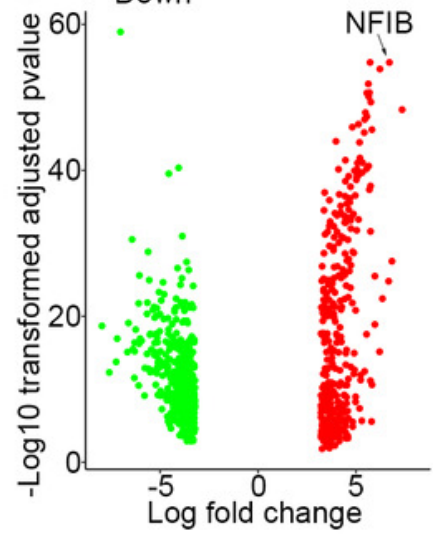

$\mathrm{F}$
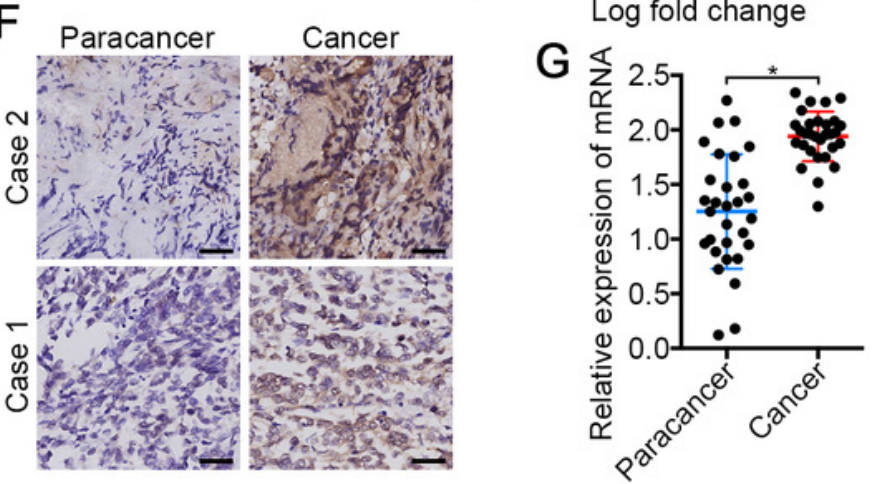

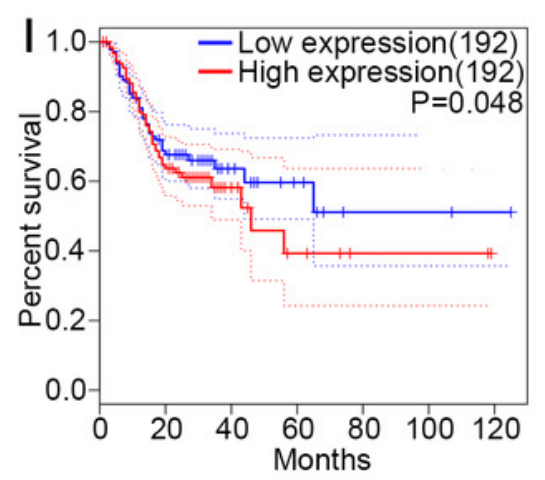




\section{Figure 2}

The high-expression of NFIB promotes the proliferation and migration of KIRC cells

(A) The promoting efficiency of NFIB vector was evaluated by RT-qPCR and western blotting in LoMet-ccRCC and 786-0 cells respectively. (B) LoMet-ccRCC and 786-0 cells were transfected with NFIB vector or negative control (NC) vector for $24 \mathrm{~h}$, and the proliferative ability was assessed by MTT assay over a 5 -day period. (C-D) Colony formation assay (1X) was performed in LoMet-cCRCC and 786-0 cells transfected with NFIB vector or NC vector for 15 days. Migration ability was assessed in LoMet-ccRCC and 786-0 cells transfected with NFIB vector or NC vector by (E-F) transwell assay, (G-H) invasion assay, and (I-J) wound healing assay $(200 x)$, Scale bars $=100 \mu \mathrm{m}$. All data were presented as means \pm SD of three independent experiments. Values are significant at $* \mathrm{P}<0.05$, as demonstrated by paired Student's t test. 

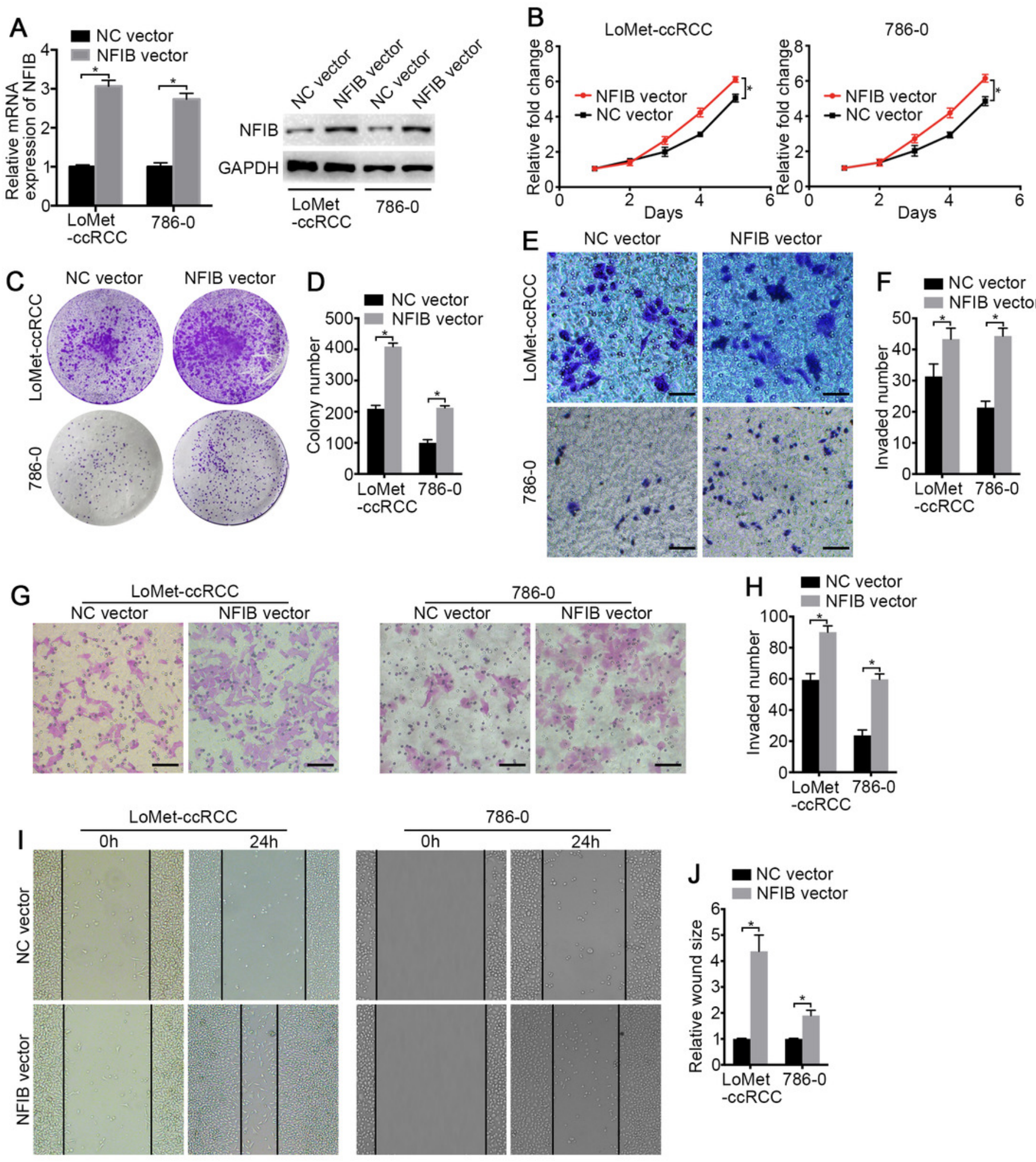


\section{Figure 3}

The low-expression of NFIB inhibits the proliferation and migration of KIRC cells

(A) The inhibiting efficiency of NFIB siRNA was evaluated by RT-qPCR and western blotting in LoMet-ccRCC and 786-0 cells respectively. (B) LoMet-ccRCC and (C) 786-0 cells were transfected with NFIB siRNA or negative control (NC) siRNA for $24 \mathrm{~h}$, and the proliferative ability was assessed by MTT assay over a 5-day period. (D-E) Colony formation assay was performed in LoMet-cCRCC and 786-0 cells transfected with NFIB siRNA or NC siRNA for 15 days. Migration ability was assessed in LoMet-ccRCC and 786-0 cells transfected with NFIB siRNA or NC siRNA by ( $F-G)$ transwell assay, $(\mathrm{H}-\mathrm{J})$ invasion assay, and (K-M) wound healing assay $(200 x)$, Scale bars $=100 \mu \mathrm{m}$. All data were presented as means \pm SD of three independent experiments. Values are significant at $* \mathrm{P}<0.05$, as demonstrated by paired Student's t test. 

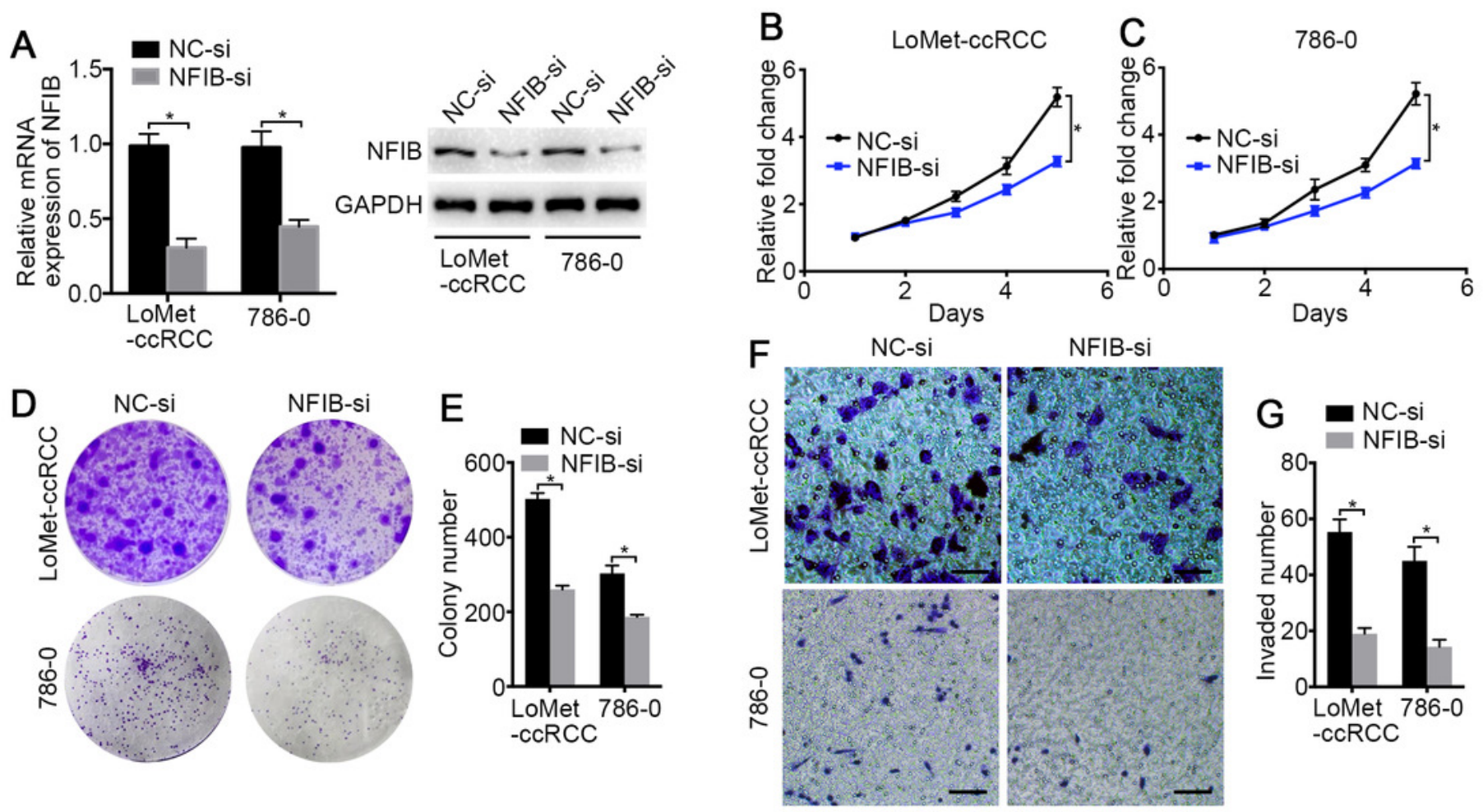

NFIB-si
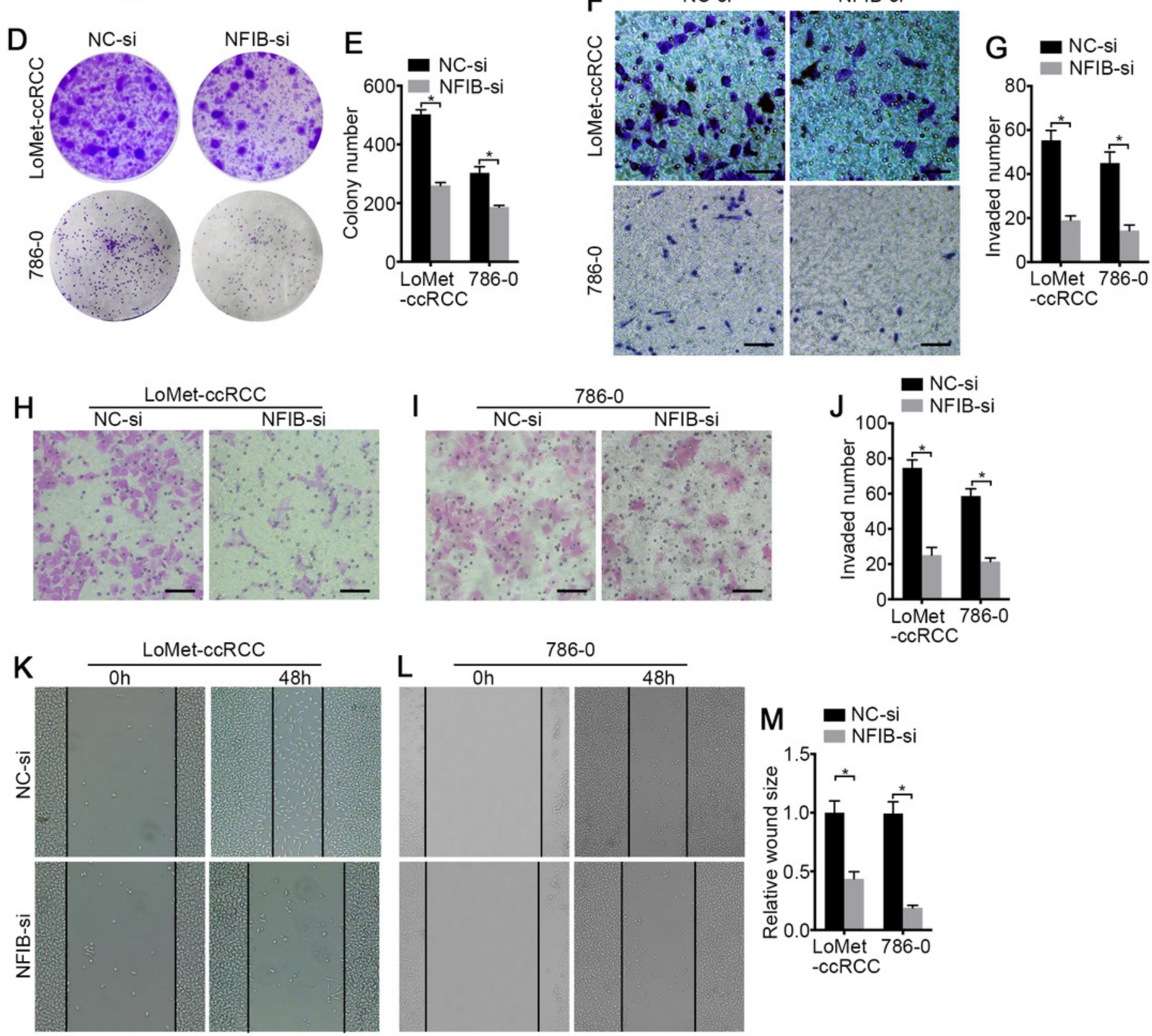


\section{Figure 4}

PINK1 was the pivotal downstream of NFIB to promote the progress of KIRC

(A) Important KEGG pathways of differentially expressed genes in three datasets. (B)

Potential binding sites of NFIB on the PINK1 gene promoter. After transfected with NFIB vector or NC vector respectively, the expression of PINK1 mRNA and protein in LoMet-ccRCC and 786-0 cells were analyzed by (C) qRT-PCR and (D) western blot assay respectively. After transfected with NFIB siRNA or NC siRNA respectively, the expression of PINK1 mRNA and protein in LoMet-ccRCC and 786-0 cells were analyzed by (E) qRT-PCR and (F) western blot assay respectively. (G) CHIP experiment was performed by using the NFIB and IgG antibodies to probe LoMet-ccRCC cells extracts, and the level of the co-precipitated RNAs were determined by using qRT-PCR. The data $\mathrm{C}, \mathrm{E}$ and $\mathrm{G}$ were presented as means $\pm \mathrm{SD}$ of three independent experiments. Values are significant at $* \mathrm{P}<0.05$, as demonstrated by paired Student's t test.

A
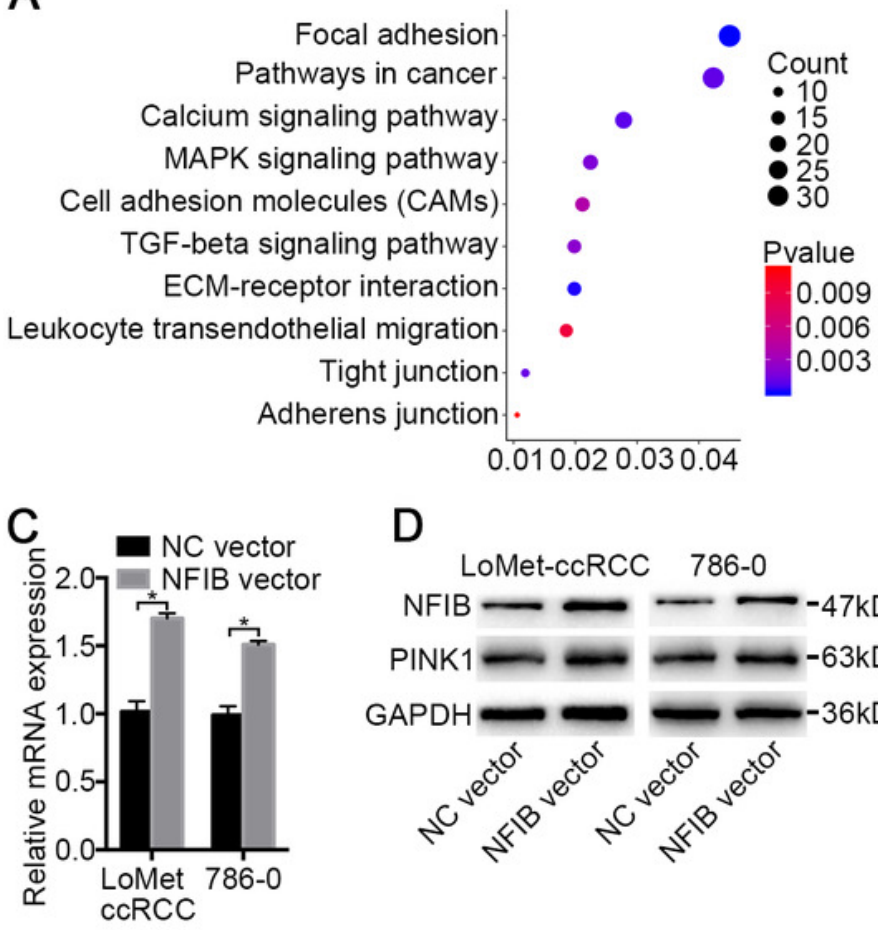

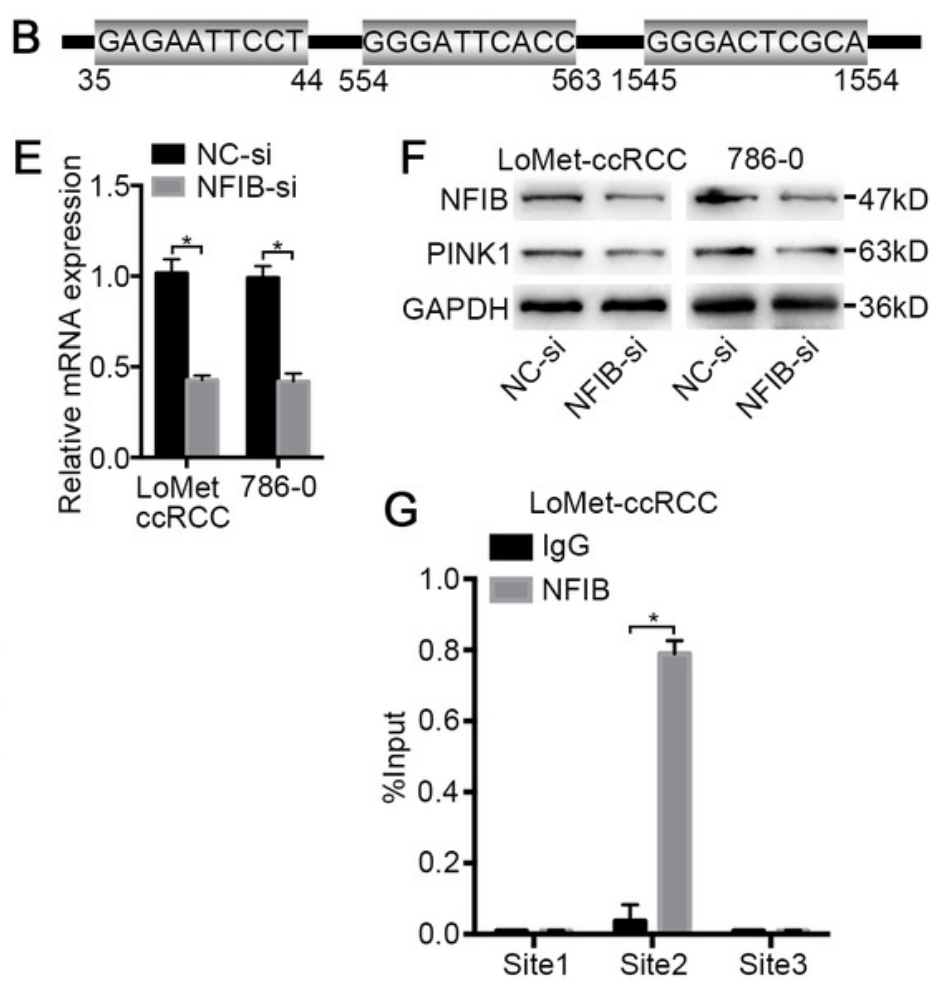




\section{Figure 5}

PINK1 knockdown inhibits the carcinogenic effects of NFIB

After co-transfection with NFIB vector and PINK1 siRNA or NC siRNA respectively, the expression of PINK1 mRNA and protein in LoMet-cCRCC and 786-0 cells were measured by (A-

B) qRT-PCR and (C) western blot assay respectively. The proliferative capacity in LoMetcCRCC cells were analyzed by (D) MTT and (E-F) colony formation assay (1X). The migration ability in LoMet-ccRCC cells were analyzed by (G-H) transwell assay, (I-J) invasion assay and $(\mathrm{K}-\mathrm{L})$ wound healing assay $(200 \times)$, Scale bars $=100 \mu \mathrm{m}$. All data were presented as means \pm SD of three independent experiments. Values are significant at $* \mathrm{P}<0.05$, as demonstrated by paired Student's t test. 


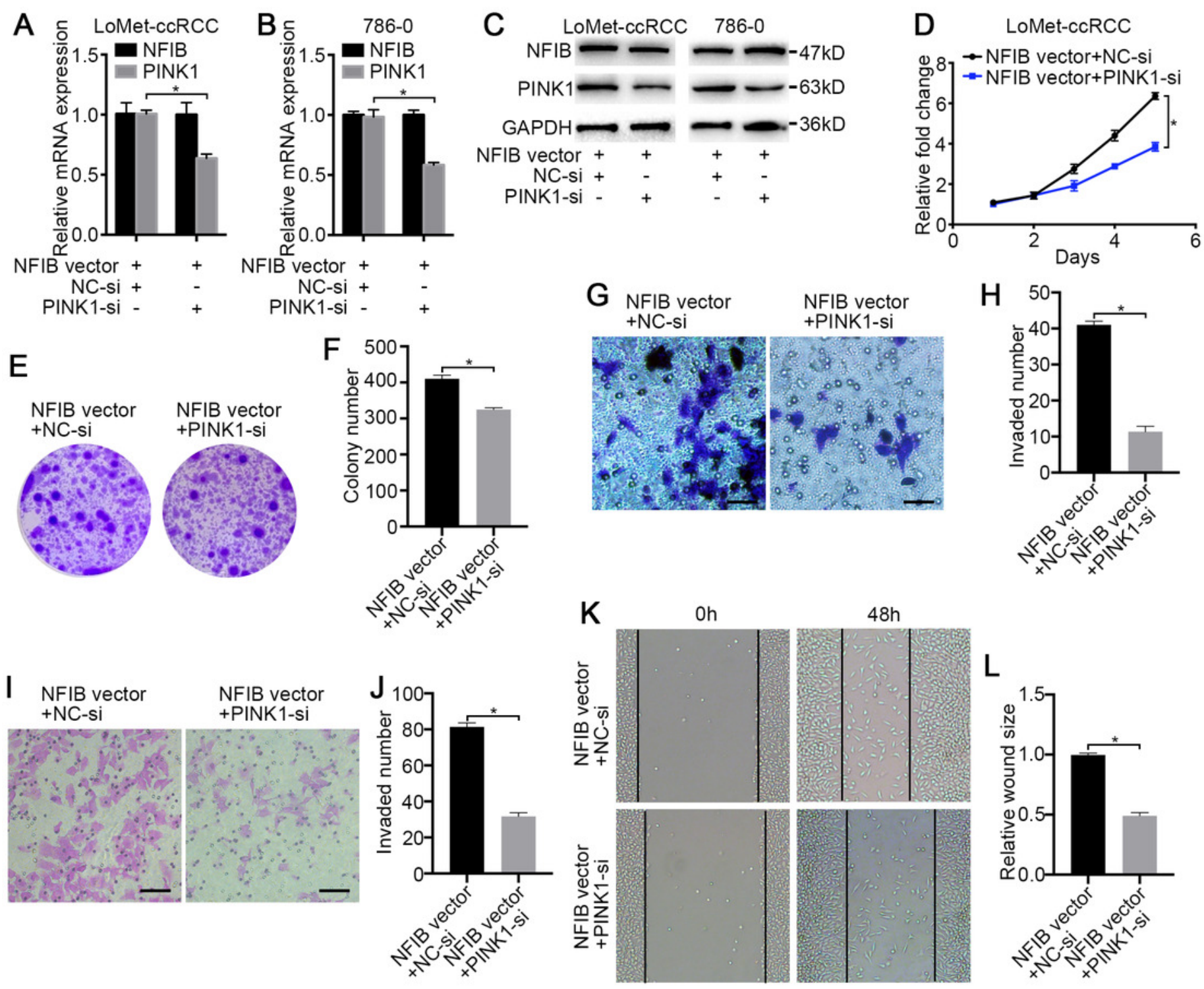




\section{Figure 6}

PINK1 is the critical downstream of NFIB in KIRC

After co-transfection with NFIB sgRNA and PINK1 vector or NC vector respectively, the expression of PINK1 protein in LoMet-cCRCC and 786-0 cells were measured by (A) western blot assay. The proliferative capacity in 786-0 cells were analyzed by (B) MTT and (C-D) colony formation assay $(1 \times)$. The migration ability in $786-0$ cells were analyzed by (E-F) transwell assay, (G-H) invasion assay and (-J) wound healing assay (200x), Scale bars $=100$ $\mu \mathrm{m}$. All data were presented as means $\pm \mathrm{SD}$ of three independent experiments. Values are significant at $* \mathrm{P}<0.05$, as demonstrated by paired Student's t test. 
A

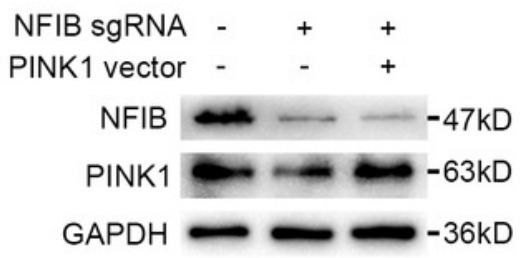

C

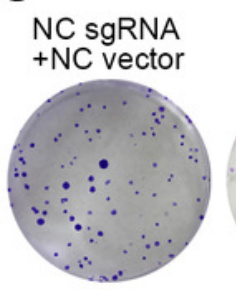

NFIB sgRNA NFIB sgRNA $+\mathrm{NC}$ vector +PINK1 vector

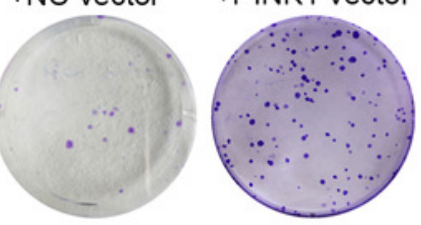

PFF

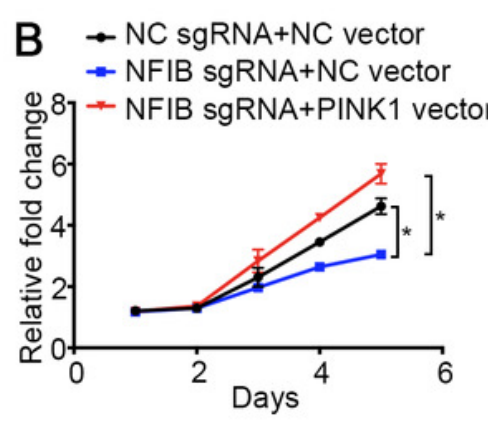

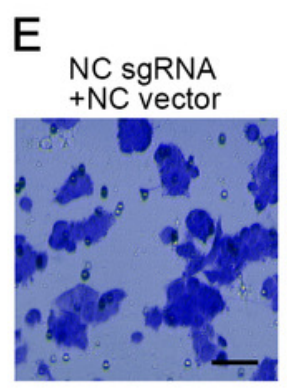
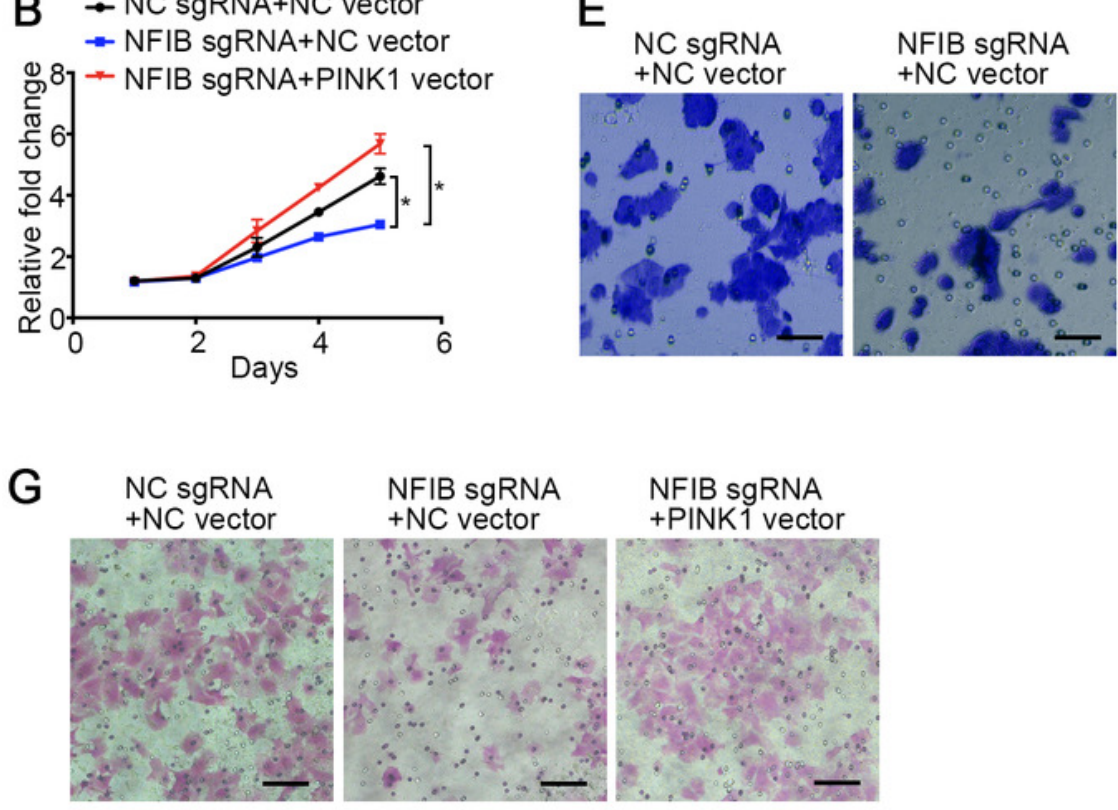

$\mathrm{H}$

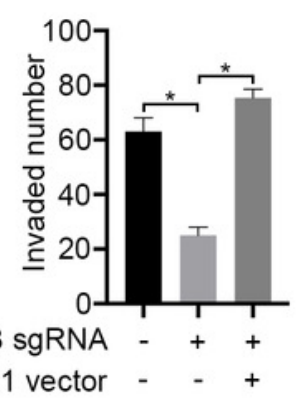

NFIB sgRNA NFIB sgRNA + NC vector +PINK1 vector

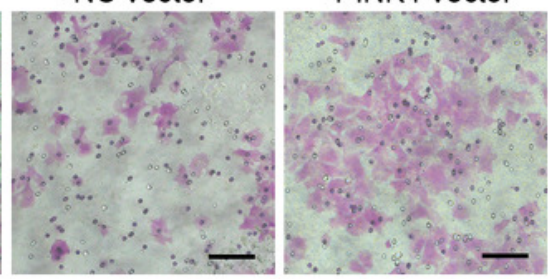

NFIB sgRNA - + +
$J$

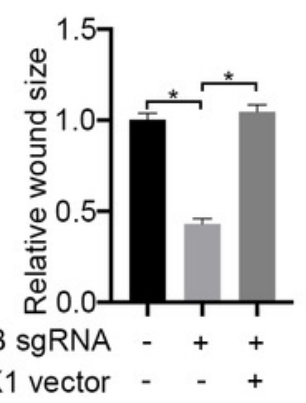

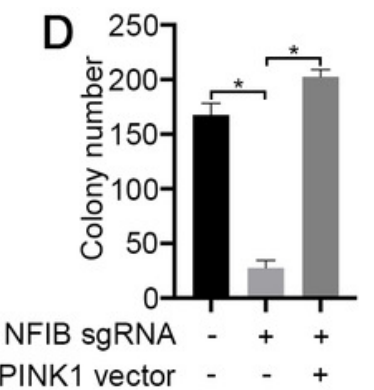

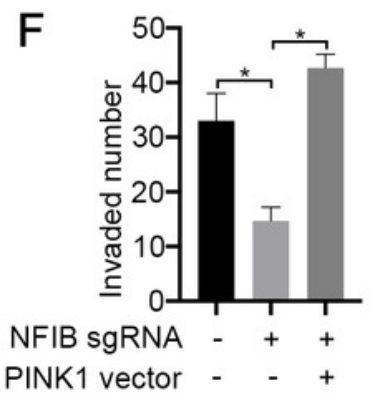

PINK1 vector - - +
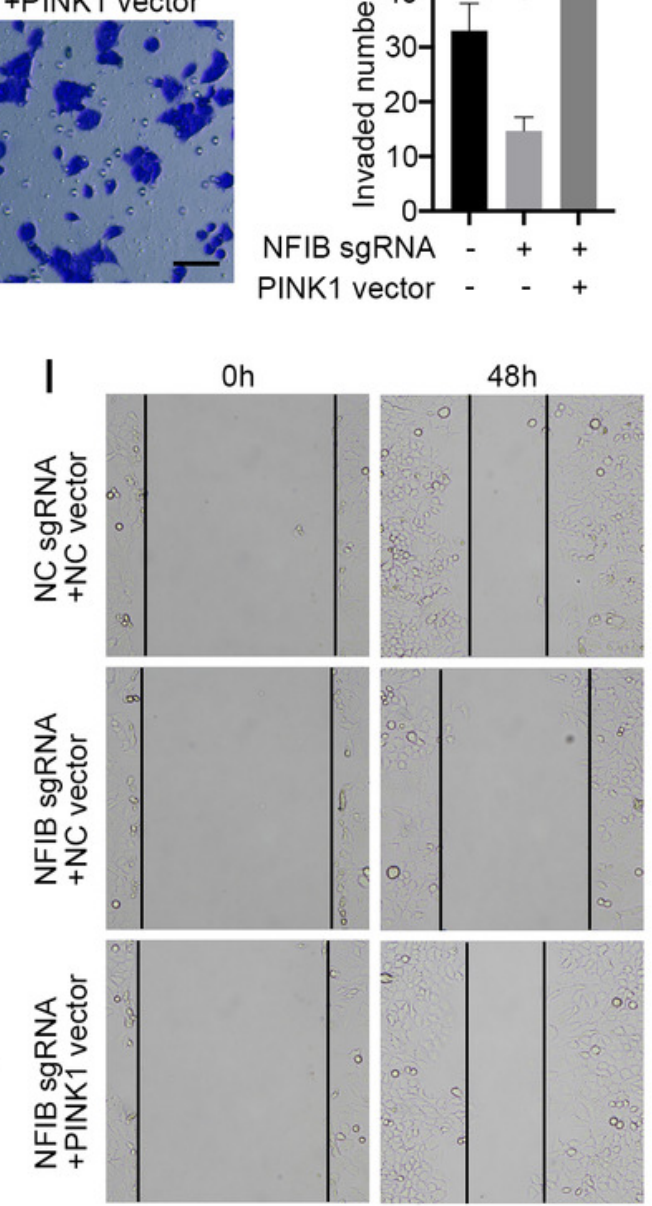


\section{Table 1 (on next page)}

Association of clinicopathological characteristics with NFIB expression 
1

\begin{tabular}{|c|c|c|c|c|}
\hline Variable & $\mathbf{n}$ & NFIB & & $\begin{array}{l}\text { Adjusted } \\
\text { P value }\end{array}$ \\
\hline & & Low expression & High expression & \\
\hline \multicolumn{5}{|l|}{ Age } \\
\hline$<60$ & 149 & 72 & 77 & 0.526 \\
\hline$>60$ & 301 & 155 & 146 & \\
\hline \multicolumn{5}{|l|}{ Sex } \\
\hline Male & 275 & 139 & 136 & 0.598 \\
\hline Female & 175 & 84 & 91 & \\
\hline \multicolumn{5}{|c|}{ Tumor laterality } \\
\hline Left & 227 & 118 & 109 & 0.368 \\
\hline Right & 220 & 105 & 115 & \\
\hline \multicolumn{5}{|c|}{ Histologic grade } \\
\hline $\mathrm{G} 1+\mathrm{G} 2$ & 280 & 157 & 123 & $0.008^{*}$ \\
\hline $\mathrm{G} 3+\mathrm{G} 4$ & 113 & 48 & 65 & \\
\hline \multicolumn{5}{|c|}{ Pathologic stage } \\
\hline $\mathrm{I}+\mathrm{II}$ & 255 & 141 & 114 & $0.011^{*}$ \\
\hline III+IV & 146 & 63 & 83 & \\
\hline \multicolumn{5}{|c|}{ Tumor stage } \\
\hline $\mathrm{T} 1+\mathrm{T} 2$ & 247 & 134 & 113 & $0.035^{*}$ \\
\hline $\mathrm{T} 3+\mathrm{T} 4$ & 168 & 75 & 93 & \\
\hline \multicolumn{5}{|c|}{ Lymph node status } \\
\hline N0 & 318 & 171 & 147 & $<0.001^{*}$ \\
\hline N1 & 126 & 44 & 82 & \\
\hline \multicolumn{5}{|c|}{ Metastasis status } \\
\hline M0 & 140 & 81 & 59 & $0.019^{*}$ \\
\hline M1 & 280 & 128 & 152 & \\
\hline
\end{tabular}

2 